\title{
Geriatric Assessment in Oncology: G8 Screening Tool Validation and Reliability Study
}

\author{
(i) Esra ATAKUL, ${ }^{3}$ (1) İmatullah AKYAR, ${ }^{1}$ (1) Deniz KOÇOĞLU TANYER ${ }^{2}$ \\ 'Department of Medical Nursing, Hacettepe University, Faculty of Nursing, Ankara-Turkey \\ ${ }^{2}$ Department of Public Health Nursing, Selçuk University, Faculty of Health Sciences, Konya-Turkey \\ ${ }^{3}$ Department of Hematology-Oncology, Health Sciences University, Dr. Abdurrahman Yurtaslan Oncology Training and Research \\ Hospital, Ankara-Turkey
}

\section{OBJECTIVE}

The present study aimed to test the validity and reliability of the Geriatrics 8 (G8) tool, a risk-screening tool for further comprehensive geriatric assessment, with hematologic cancer patients aged 65 years and older.

\section{METHODS}

This methodological study included 110 patients followed up for hematologic cancer. Data were collected using the patient identification form, the G8 screening tool, and the Edmonton Frail Scale. Language and content validity, internal consistency, confirmatory factor, sensitivity and specificity and concurrent validity analyses were also used.

\section{RESULTS}

Patients (mean age: $73.9 \pm 6.10)$ were diagnosed with lymphoma (30.9\%), multiple myeloma $(21.8 \%)$, leukemia (20.9\%), myelodysplastic syndrome (19.1\%). Confirmatory factor model fit index analysis $\chi^{2} /$ $\mathrm{df}$ and $\mathrm{p}$-values (0.14) showed a good fit and GFI, AGFI, SRMR, and RMSEA values showed acceptable fit. The Cronbach's alpha coefficient was 0.655 , area under the ROC curve was 0.824 (95\%, 0.745-0.904), and sensitivity and specificity were $61.0 \%$ and $88.9 \%$, respectively.

\section{CONCLUSION}

The findings indicated that the G8 screening tool was found a valid and reliable tool for geriatric patients diagnosed with cancer to determine the risk and need for further comprehensive geriatric assessment.

Keywords: Aged; frail elderly; geriatric assessment; geriatric nursing/methods; hematology; risk factors. Copyright $\odot$ 2019, Turkish Society for Radiation Oncology

\section{Introduction}

Older adults are among the fastest-growing age group of the population and by 2030 are expected to represent $20 \%$ and $30 \%$ of the US and European populations, respectively.[1] Approximately $70 \%$ of the cancer deaths and $60 \%$ of all new cancer cases occur in adults aged 65 years and older, and cancer incidence is 11 times higher than the younger population. [2,3] Although the number of cases increasing, standards and guidelines for routine care for older adults diagnosed with cancer are limited, and this group is not adequately represented in clinical trials.[4]

Older adults are different from each other regarding physiological, psychological, social health, functionality, independence level, comorbidities, and cultural aspects. The differences in the aging process, combined with heterogeneity in health, functional, 
and social status between individuals or within an individual timeline, lead to differences in treatment and care. The limited representation of older adults in clinical trials adds challenges in oncology care. [5] As a result, the complexity and uniqueness of older adults diagnosed with cancer urge to provide evidence-based planned treatment and care.[4-6] Commonly, to guide the provision of evidence-based treatment and care, and to assess the adult and multifactorial process of aging comprehensive geriatric assessment (CGA) is used. The CGA is an interdisciplinary approach where multidimensional medical, functional, psychosocial, and environmental assessments are conducted to develop a coordinated and long-term plan for the diagnosis and treatment of elderly individuals. [7-9]

The CGA approach has been used in the care of older adults diagnosed with cancer since the 1990s. [10] The Geriatric Oncology of the National Comprehensive Cancer Network, the International Society of Geriatric Oncology, and the European Organization for Research and Treatment of Cancer also advised utilizing CGA in the care of the older adults diagnosed with cancer.[4,11-13] The CGA found to be useful in determining prognosis, risks, and treatment benefits and laying the groundwork for interventions to improve patient outcomes. $[4,14]$ Previous studies showed that individualized care developed through CGA was helpful in achieving maximum treatment efficacy and minimum toxicity; whereas non-CGA based care of older adults diagnosed with cancer resulted in disruptions. [15,16]

Deschodt et al.[17] (2013) reported that the multidisciplinary CGA approach has three stages: 1) case finding or screening (identifying high-risk groups), 2) assessment (comprehensive assessment), and 3) implementation (evidence-based care based on need). To include CGA in routine care, which is a standard of care in oncology practice, older adults diagnosed with cancer must be screened to check whether full CGA is required or not.[18,19] Given the time and resource barriers related to CGA, the use of geriatric screening tool become prominent; $[5,20,21]$ multiple screening tools, such as abbreviated Comprehensive Geriatric Assessment,[22] Cancer-Specific Geriatric Assessment instrument,[23] Vulnerable Elderly Survey-13,[24] Fried Frailty Criteria, [5,21] and Geriatrics 8 (G8).[25] Although the choice of the tool depends on clinical resources, G8 was recommended by SIOG with its highest sensitivity.[25-27] This study aimed to investigate the psychometric properties and screening performance of the G8 screening tool, with geriatric hematologic patients aged 65 years and older.

\section{Materials and Methods}

\section{Study Design}

This methodological study was conducted to investigate the validity and reliability, and psychometric properties of the Turkish version of the "G8 screening tool", used to evaluate geriatric risk profiles of patients over 65 years of age diagnosed with hematologic cancer.

\section{Setting and Sample}

The study population consisted of hematologic cancer patients aged 65 years and older who were admitted to hematology outpatient clinics of a state hospital. Study samples constituted as representing at least 10 patients per item of the scale. A total of 110 patients were included in this study to increase the representational power. Patients aged 65 years and older, diagnosed with hematologic cancer, literate, without communication problems and cognitive disorders (advanced Alzheimer's disease and dementia), and provided informed consent were included in this study.

\section{Data Collection}

Data were collected from 110 patients who were admitted at a state hospital between May $4^{\text {th }}$-February $20^{\text {th }}$, 2017. The patients admitted to the outpatient clinic were approached after their visit, while the patients in inpatient units were approached before the morning rounds. Patients were provided with information before this study, and informed consent was obtained from each patient. The researchers conducted face-toface interviews to collect data for this study. Data collection lasted about 10 minutes per participant.

\section{Data Collection Tools}

Data were collected using patient identification form, the G8 screening tool and the Edmonton Frail Scale.

Patient identification form consists of 16 questions about the socio-demographic characteristics of patients and disease characteristics.

The G8 screening tool was developed by Soubeyran et al. in 2012, to assess the need for CGA in older adults diagnosed with cancer. The tool was created by adding the item "age" to the seven items selected from the Mini Nutritional Assessment scale. G8 selects the groups that need further comprehensive assessment. Among the items selected from the Mini Nutritional 
Assessment scale are nutritional status, weight loss, body mass index, number of medications used, and perceived health. Age is considered in three categories ( $<80$ years, $80-85$ years, and $>85$ years). The G8 score ranges from 0 to 17 . The items are interpreted using the scoring values in the Mini Nutritional Assessment scale. Scores $\leq 14$ indicate abnormal. In addition to nutritional status, the G8 screening tool produces results about CGA areas, such as patients' comorbidity, mental distress, and cognitive status.[25,28]

Edmonton Frail Scale was developed by Rolfson et al.[29] (2006) in Canada to assess frailty in older adults. The Cronbach alpha coefficient of the original scale is 0.62 . The reliability and validity test of the scale in Turkey was carried out by Aygor \& Fadiloglu. The number of items in the scale was not changed while it was being adapted to the Turkish population, and the Cronbach alpha of the scale was reported to be 0.75.[30] The scale consists of nine frailty domains included in the CGA and considered to be determinants of frailty. These domains are cognition, functional performance, general health status, functional independence, social support, pharmacological condition, nutritional aspect, mental condition, and continence. The scale consists of 11 items. General health status and pharmacological conditions are individually assessed with two questions, while the other domains are individually assessed with a single question. Cognition and functional independence are assessed using the "clock" and "timed up and go" tests, respectively. The questions are scored 0,1 , or 2 . Scale concludes as not frail (scored 0-4), and frail as apparently vulnerable (scored 5-6), mildly frail (scored 7-8), moderate frailty (scored 9-10), and severe frailty (scored $>11$ ).[24] In this study, the Edmonton Frail Scale was used to assess the sensitivity and specificity of the G8 screening tool.

\section{Ethical Considerations}

Permission to use and adaptation of G8 screening tool obtained from investigators (Dr. P.S), and this study was approved by the Ethical Committee for Non-Interventional Clinical Investigations of the university (\# GO 15/488-27). Written consent was provided by the hospital's administration. All participants signed a written consent form.

\section{Data Analysis}

Data were analyzed using IBM Statistical Package for Social Sciences, v.23. Language validity analysis, content validity analysis, sensitivity and specificity analysis, and concurrent validity (ROC curve analysis) were performed for the Turkish adaptation of the scale. Descriptive statistics (mean, standard deviation, median, minimum, maximum, number, and percentile) were used to describe the categorical and continuous variables.

\section{Results}

\section{Study Group}

The mean age of participants was 73.9 \pm 6.10 (range 6592) years, $51.7 \%$ were men, $52.7 \%$ had a primary school degree, $80.9 \%$ were married, $50.5 \%$ had 4 or more children, $70.9 \%$ lived with their children, and $61.8 \%$ described their economic status as "expenses equal income". The participants were diagnosed with lymphoma (30.9\%), multiple myeloma (21.8\%), leukemia (20.9\%), myelodysplastic syndrome (19.1\%), leukocyte disorder (6.4\%), and aplastic anemia (grouping based on WHO 2001 classification of hematological malignancies). Approximately $70 \%$ of the patients had at least one chronic disease. The duration of follow-up was $3.05 \pm 1.56$ (max: 9 ) years.

\section{Validity Results}

Language Validity Analysis: The tool was translated into Turkish by three faculty members (two nursing faculty, one physician). Three translations gathered into a single version by the researchers. The tool was back-translated (Turkish to English) by three experts (nursing faculty, physician, and interpreter). The backtranslated version was compared with the original tool and found to be consistent. An expert Turkish linguist checked the final version for grammar, spelling, and punctuation.

Content Validity Analysis: Content validity analysis was performed by experts to determine whether the items represent and cover the concept to be measured, as well as the comprehensibility. The tool was evaluated by five experts from internal medicine, hematology, nutrition and dietetics and geriatrics fields. The experts rated each item on the scale according to Davis technique (1: not appropriate, 2: slightly appropriate, 3: appropriate, 4: very appropriate). The items were re-evaluated with expert evaluations, the content validity indices calculated according to the ratings from experts. The content validity indices of the scale items calculated, and the content validity index for each item was found to be 1 .

Construct Validity and Internal Consistency Analysis: Confirmatory factor analysis used to test the construct validity of the G8 screening tool. Confirma- 
tory factor model fit index analysis, $\chi^{2} / \mathrm{sd}$ and $\mathrm{p}$-values (0.14) showed a good fit and GFI, AGFI, SRMR, and RMSEA values showed acceptable fit. The CFI was slightly below the acceptable fit, but it was accept-

\begin{tabular}{lccc} 
Table 1 & \multicolumn{3}{c}{ Confirmatory factor model fit index analysis } \\
Fit Index & Good fit & Acceptable fit & Study result \\
\hline $\mathrm{X}^{2} / \mathrm{df}$ & $0 \leq \mathrm{X}^{2} / \mathrm{sd} \leq 3$ & $3 \leq \mathrm{X}^{2} / \mathrm{sd} \leq 5$ & 1.33 \\
$\mathrm{GFI} \geq 0.95$ & $\geq 0.90$ & 0.94 & \\
$\mathrm{AGFI}$ & $\geq 0.95$ & $\geq 0.90$ & 0.89 \\
$\mathrm{CFI} \geq 0.97$ & $\geq 0.95$ & 0.92 & \\
$\mathrm{SRMR}$ & $\leq 0.05$ & $0.06-0.08$ & 0.07 \\
RMSEA & $\leq 0.05$ & $0.06-0.08$ & 0.05 \\
\hline
\end{tabular}

df: Degree of Freedom; GFI: Goodness of Fit Index; AGFI: Adjusted Goodness of Fit Index; CFI: Comparative Fit Index; SRMR: Standardized Root-MeanSquare Residual; RMSEA: Root-Mean-Square Error of Approximation.

Table 2 Cronbach's alpha coefficient

\begin{tabular}{ccc} 
& Cronbach alfa coefficient & Split half \\
\hline G8 screening tool & 0.655 & $\mathrm{r}: 0.60$ \\
& & $\mathrm{p}<0.001$ \\
\hline
\end{tabular}

Table 3 G8 total score item correlations

\begin{tabular}{lcc} 
Items & $\begin{array}{c}\text { Corrected item - total } \\
\text { score correlation }\end{array}$ & $\begin{array}{c}\text { Item deletion } \\
\text { Alpha coefficient }\end{array}$ \\
\hline Item 1 & 0.605 & 0.409 \\
Item 2 & 0.420 & 0.487 \\
Item 3 & 0.411 & 0.504 \\
Item 4 & 0.442 & 0.550 \\
Item 5 & 0.390 & 0.557 \\
Item 6 & 0.313 & 0.571 \\
Item 7 & 0.324 & 0.513 \\
Item 8 & 0.016 & 0.603 \\
\hline
\end{tabular}

Table $4 \quad$ ROC analysis

\begin{tabular}{lcc} 
Variable & $\begin{array}{c}\text { Area, } \\
\text { p-value }\end{array}$ & $\begin{array}{c}\text { \%95 Confidence } \\
\text { interval } \\
\text { Min-Max }\end{array}$ \\
\hline G8 score & 0.824 & $0.745-0.904$ \\
\hline Cut-off & 0.000 & 1- Specificity \\
\hline$>13.5$ & Sensitivity & 73.3 \\
$>14.5$ & 72.0 & 88.9 \\
$>15.5$ & 61.0 & 95.6 \\
\hline
\end{tabular}

able since it was greater than 0.90. Model fit indices showed compliance of the measurement model as sufficient and, therefore, the G8 screening tool was valid (Table 1). The reliability of the G8 tool was assessed using Cronbach's alpha and half-divide method. The Cronbach's alpha coefficient and Spearman-Brown coefficient were calculated as 0.655 and 0.60 , respectively (Tables 2, 3). G8 tool was found to be quite reliable.

\section{Reliability Results}

Concurrent validity (Sensitivity and Specificity Analysis): The Edmonton Frail Scale was used to test the concurrent validity of the G8 screening tool. Sensitivity measurement was used to determine the group requiring further comprehensive assessment (scored abnormal) while specificity measurement is used to recognize those who are normal within the normal group (Table 4). The area under the G8 screening tool's total score was 0.824 , and the measurement power was "good" (Fig. 1). The sensitivity and specificity were $61.0 \%$ and $88.9 \%$, respectively.

\section{Discussion}

Geriatric care focuses on quality of life and functionality, with an individualized, holistic approach. Cancer diagnosis in older adults makes treatment and care more complicated due to comorbidities and additional

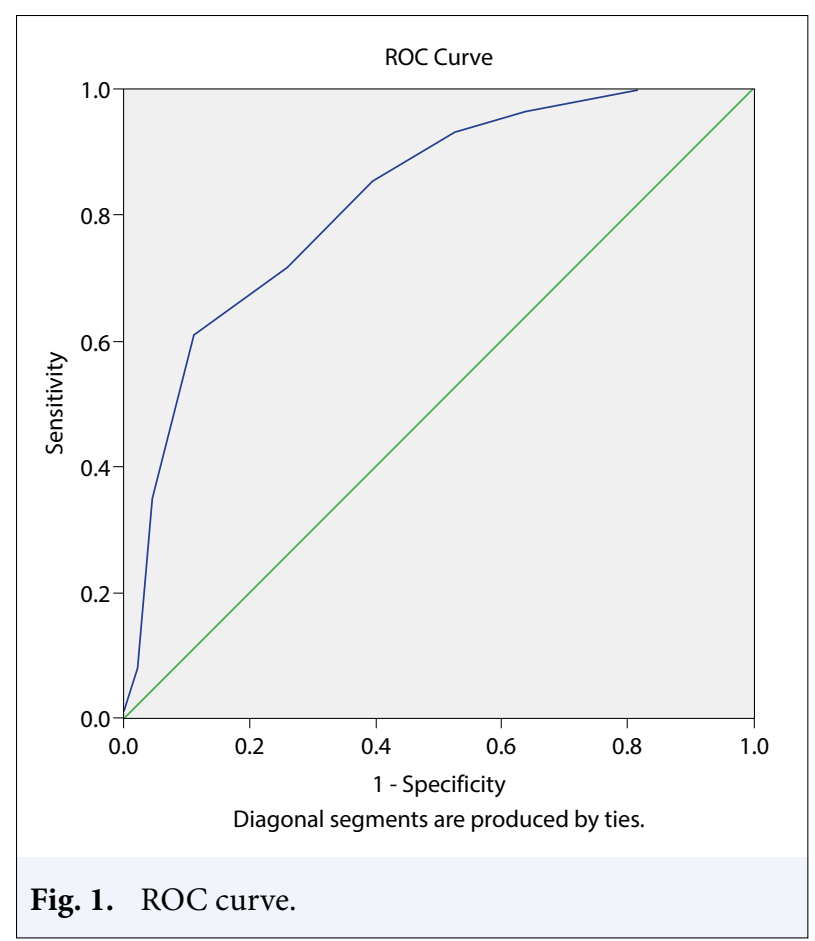


geriatric problems, which urges individual treatment and care. CGA ensures that the risks of side effects are assessed, geriatric conditions are identified, and diagnosis and multidisciplinary interventions are used to obtain positive health outcomes in older adults. Geriatric assessment, which helps to identify effective interventions, is very important in improving function, quality of life, and individualized treatment and care. Many studies have been conducted to evaluate the screening tools since the International Society of Geriatric Oncology guidelines emphasized in 2005 the need for risk screening of older adults with cancer. $[31,32]$ The Turkish version of the G8 screening tool, the most widely used tool, was adapted in this study to investigate the need for the CGA of older adults diagnosed with hematologic cancer.

The adaptation of the G8 screening tool to Turkish began with language validity and content validity analysis based on expert opinion. Confirmatory factor analysis, half-divide method, sensitivity and specificity analysis, and ROC curve analysis were performed to determine the validity of the scale. The validity and reliability analysis of the tool showed that the G8 screening tool had acceptable validity, good fit, and good reliability. The screening tool was applied by an oncology nurse to outpatients and inpatients in about five minutes each. The G8 screening tool can be applied to older outpatients and inpatients quickly and easily in intense work environments where time and resources are limited.

ROC analysis was performed for sensitivity and specificity analysis. In the original study, the G8 screening tool had a cut-off point of $\leq 14$, a sensitivity of $85 \%$, and a specificity of $65 \%$.[25] According to the systematic review of Decoster et al., the G8 screening tool was used in eight studies (3.816 patients), with a sensitivity ranging from $65 \%$ to $92 \%$ (over $80 \%$ in 6 studies) and a specificity ranging from $3 \%$ to $75 \%$ (over 60\% in 4 studies). Velghe et al. (2014) conducted a study on elderly individuals diagnosed with aggressive hematologic cancer and their findings showed that the sensitivity and specificity of the tool to be $89 \%$ and $100 \%$, respectively.[33] In this study, the cut-off point, sensitivity, and specificity of the 17-point scale were found as $14.5,61.0 \%$, and $88.9 \%$, respectively.

According to the Edmonton Frail Scale, $41.3 \%$ of the participants were considered frail (median scale: 6 , mean: $6.06 \pm 3.25)$. According to the G8 screening tool, $59.1 \%$ of the participants constituted the further need group for CGA $(<14.5)$. The G8 median score was 14.5, while the mean score was $13.21 \pm 2.82$ (range 4-17). In the original study in which the G8 screening tool was developed, the score ranged from 6.5 to 17 , and the median was 12.[25]

\section{Limitations of this Study}

This study had some limitations. First, the general health status of participants was found to be similar, and they were monitored with a specific treatment policy in the same institution. However, this study was conducted with patients from outpatient and inpatient units. Therefore, this should be considered in the implementation of the results. Second, the measurements were performed only once, and no further evaluation was conducted with repeated measurements.

\section{Conclusion}

Geriatric assessment in oncology settings can help to determine the vulnerability, and functionality, predict toxicity and survival, help in clinical decision-making, treatment and care plan.[34] The results obtained from the analysis of the tool translated into Turkish agree well with the findings reported in the previous studies. In this study, our results show that the G8 is a useful tool for older adults diagnosed with hematologic cancer. We believe that the G8 can be used to determine further CGA needs. Therefore, we recommend studies to assess the psychometric properties of the G8 screening tool for healthy outpatients aged 65 years and older.

Peer-review: Externally peer-reviewed.

Conflict of Interest: None declared.

Financial Support: None declared.

Authorship contributions: Concept - E.A., İ.A.; Design - E.A., İ.A.; Supervision - İ.A., D.T.; Materials - E.A.; Data collection \&/or processing - E.A.; Analysis and/or interpretation - D.T., İ.A.; Literature search - İ.A., E.A.; Writing İ.A., E.A., D.T.; Critical review - İ.A., D.T.

\section{References}

1. Federal Interagency forum on Aging Related Statistics. Older Americans 2000: key indicators of well-being. 2002. Washington DC. Access: [https://agingstats. gov/docs/PastReports/2000/OA2000.pdf]. Accessed 22 April 2019.

2. Yancik R. Cancer burden in the aged: an epidemiologic and demographic overview. Cancer 1997;80(7):127383.

3. Berger NA, Savvides P, Koroukian SM, Kahana EF, Deimling GT, Rose JH, et al. Cancer in the elderly. Trans Am Clin Climatol Assoc 2006;117:147-55. 
4. Given B, Given CW. Older adults and cancer treatment. Cancer 2008;113(12 suppl): 3505-11.

5. Loh KP, Soto-Perez-de-Celis E, Hsu T, de Glas NA, Battisti NM, Baldini C, et al. What every oncologist should know about geriatric assessment for older patients with cancer: young international society of geriatric oncology position paper. J Oncol Pract. 2018;14(2):85-94.

6. Akdemir N, Akyar I. Geriatric nursing. J Acad Geriatr 2009;1(2):73-81.

7. Rubenstein LZ, Stuck AE, Siu AL, Wieland D. Impacts of geriatric evaluation and management programs on defined outcomes: overview of the evidence. J Am Geriatr Soc 1991;39(9 Pt 2):8-16.

8. Kane RL. Choosing and Using and Assessment Tool. In: Kane RL, Kane RA, editors. Assessing Older Persons: Measures, Meaning, and Practical Applications. 1st Edition. UK: Oxford University Press; 2004. p. 237-60.

9. Gallo J, Bogner HR. Multidimensional assessment. In: Gallo JJ, Bogner HR, Fulmer T, Paveza GJ, editors. Handbook of Geriatric Assessment. 4th Edition. USA: Jones \& Bartlett Publishers; 2006. p. 6-11.

10. Extermann M, Aapro M, Bernabei R, Cohen HJ, Droz JP, Lichtman S, et al. Task Force on CGA of the International Society of Geriatric Oncology. Use of comprehensive geriatric assessment in older cancer patients: Recommendations from the task force on CGA of the International Society of Geriatric Oncology (SIOG). Crit Rev Oncol Hematol 2005;55(3):241-52.

11. Droz JP, Balducci L, Bolla M, Emberton M, Fitzpatrick JM, Joniau S, et al. Management of prostate cancer in older men: recommendations of a working group of the International Society of Geriatric Oncology. BJU Int 2010;106(4):462-9.

12. NCCN Clinical Practice Guidelines in Oncology Senior Adult Oncology Version 2. 2011 [Internet]. National Comprehensive Cancer Network. [cited 2019 April 22]. Available at: http://www.nccn.org/professionals/physician_gls/pdf/senior.pdf. Accessed 2019, Oct 2.

13. International Society of Geriatric Oncology.Comprehensive Geriatric Assessment (CGA) of the older patient with cancer. SIOG [cited 2019 April 22]. Available from: http://www.siog.org/content/comprehensive-geriatric-assessment-cga-older-patientcancer.

14. Owusu C, Berger NA. Comprehensive geriatric assessment in the older cancer patient: coming of age in clinical cancer care. Clin Pract (Lond) 2014;11(6):749-62.

15. Yancik R, Ries LA. Cancer in older persons: an international issue in an aging world. Semin Oncol 2004;31(2):128-36.

16. Kalsi T, Babic-Illman G, Ross PJ, Maisey NR, Hughes S, Fields P, et al. The impact of compre- hensive geriatric assessment interventions on tolerance to chemotherapy in older people. Br J Cancer 2015;112(9):1435-44.

17. Deschodt M, de Casterle BD, Milisen K. Gerontological care in nursing education programmes. J Adv Nurs 2010;66(1):139-48.

18. Puts MT, Hardt J, Monette J, Girre V, Springall E, Alibhai SM. Use of geriatric assessment for older adults in the oncology setting: a systematic review. J Natl Cancer Inst 2012;104(15):1133-63.

19. Kenis C, Decoster L, Van Puyvelde K, De Greve J, Conings G, Milisen K, et al. Performance of two geriatric screening tools in older patients with cancer. J Clin Oncol 2014;32(1):19-26.

20. Shahrokni A, Kim SJ, Bosl GJ, Korc-Grodzicki B. How we care for an older patient with cancer. J Oncol Pract 2017;13(2):95-102.

21. Marosi C, Köller M. Challenge of cancer in the elderly. ESMO Open 2016;1(3):e000020.

22. Overcash JA, Beckstead J, Moody L, Extermann M, Cobb S. The abbreviated comprehensive geriatric assessment (aCGA) for use in the older cancer patient as a prescreen: scoring and interpretation. Crit Rev Oncol Hematol 2006;59(3):205-10.

23. Hurria A, Gupta S, Zauderer M, Zuckerman EL, Cohen $\mathrm{HJ}$, Muss $\mathrm{H}$, et al. Developing a cancer-specific geriatric assessment: A feasibility study. Cancer J 2005;104(9):1998-2005.

24. Hentschel L, Rentsch A, Lenz F, Hornemann B, Schmitt J, Baumann $\mathrm{M}$, et al. A questionnaire study to assess the value of the vulnerable elders survey, G8, and predictors of toxicity as screening tools for frailty and toxicity in geriatric cancer patients. Oncol Res Treat 2016;39(4):210-6.

25. Soubeyran P, Bellera C, Goyard J, Heitz D, Cure H, Rousselot $\mathrm{H}$, et al. Validation of the G8 screening tool in geriatric oncology: The ONCODAGE project. J Clin Oncol 2011;29(15 suppl):9001.

26. Bostankolu Ö, Öztürk B, Coşkun U, Büyükberber S, Benekli M. Cancer chemotherapy in elderly patients. Int J Hematol Oncol 2008;18(3):186-92.

27. Martinez-Tapia C, Canoui-Poitrine F, Bastuji-Garin S, Soubeyran P, Mathoulin-Pelissier S, Tournigand $\mathrm{C}$, et al. Optimizing the G8 screening tool for older patients with cancer: diagnostic performance and validation of a six-item version. Oncologist 2016;21(2):188-95.

28. Bellera C, Rainfray M, Mathoulin-Pelissier S, Martens C, Delva F, Fonck, M, et al. Screening older cancer patients: first evaluation of the G-8 geriatric screening tool. Ann Oncol 2012; 23(8):2166-72.

29. Rolfson DB, Majumdar SR, Tsuyuki RT, Tahir A, Rockwood K. Validity and reliability of the Edmonton Frail Scale. Age Ageing 2006;35(5):526-9. 
30. Aygör HE, Fadıloğlu Ç, Şahin S, Aykar FŞ, Akçiçek F. Validation of Edmonton Frail Scale into elderly Turkish population. Arch Gerontol Geriatr 2018;76:133-7.

31. Decoster L, Van Puyvelde K, Mohile S, Wedding U, Basso U, Colloca G, et al. Screening tools for multidimensional health problems warranting a geriatric assessment in older cancer patients: an update on SIOG recommendations. Ann Oncol 2015;26(2):288-300.

32. Balducci L, Beghe C. The application of the principles of geriatrics to the management of the older person with cancer. Crit Rev Oncol Hematol 2000;35(3):147-54.

33. Velghe A, Petrovic M, De Buyser S, Demuynck R, Noens L. Validation of the G8 screening tool in older patients with aggressive haematological malignancies. Eur J Oncol Nurs 2014;18(6):645-8.

34. Hamaker ME, Schiphorst AH, ten Bokkel Huinink D, Schaar C, van Munster BC. The effect of a geriatric evaluation on treatment decisions for older cancer patients-a systematic review. Acta Oncol 2014;53(3):289-96. 